\title{
Sickness presenteeism in Norway and Sweden
}

\author{
Vegard Johansen
}

Eastern Norway Research Institute

Lillehammer, Norway

Email: vj@ostforsk.no

\begin{abstract}
Introduction: Sickness presenteeism (SP) refers to the practice of going to work despite illness. This article describes the distribution of SP in Norway and Sweden. It also discusses relations between $S P$ and various work characteristics and personal factors in the two countries.

Methods: More than 2500 Norwegian and Swedish workers between 20 and 60 years of age answered a postal questionnaire. The Norwegian and Swedish samples are weighed and representative with regard to both variables of regional background and demography, but the response rate was low. The distribution of SP is measured by frequency (episodes in the previous year) and by length (total days of SP in the previous year). This study employed binary and multinomial logistic regression to detect which factors influence the frequency of SP.

Results: Fifty-five per cent of the respondents in Norway and Sweden practised SP in the previous year. The frequency of SP episodes is similar in the two countries. Further, respondents with low/medium income, physical work, and managerial responsibilities report SP more often in both countries. Non-western immigrants, the less educated, and those employed by others are overrepresented with $S P$ in Norway. Neither gender nor age had any particular influence.

Discussion: In accordance with previous studies, this study among Norwegian and Swedish workers suggests that some SP during a working year may be more common than no SP. Our analyses of determinants of SP present some previously undocumented differences. Divisions between sedentary versus physical work and management versus non-management were important for $S P$ in Norway and Sweden. Moreover, non-western immigrants are overrepresented with $S P$ in Norway, but this pattern does not prevail in Sweden. Some possible causes for non-western immigrants to report more SP are suggested in the article, but we need more research to follow up on the missing correlation between ethnic background and SP in Sweden.
\end{abstract}

Keywords: sickness presenteeism, survey, Norway, Sweden, logistic regression 


\section{Introduction}

Sickness presenteeism (SP) refers to the practice of going to work despite illness. This concept has been a subject of steadily increasing interest since it emerged in the 1990s, though the number of publications on the topic is clearly fewer than those focusing on sickness absence (SA) (Vingård et al., 2004; Hansen \& Andersen, 2009; Böckerman \& Laukkanen, 2010). Using survey data from a study conducted with more than 2500 workers in Norway and Sweden, this article describes the distribution of SP and discusses some important determinants for SP.

Comparative studies are useful both for the similarities and the differences they explore. From an international perspective, one could understand this study on SP in Norway and Sweden as a comparison of most-similar cases. The chosen countries are neighbours, exchange labour extensively, have healthy populations, and are highly developed welfare systems. At the same time, sickness benefits are much more generous in Norway than they are in Sweden: a sick-listed person in Norway receives full compensation from the first day for a maximum of 364 days, whilst in Sweden the employees themselves pay for the starting day and receive 80 per cent compensation for a maximum of 364 days within a frame of 450 days (Ministry of labour and social inclusion, 2011; Government proposition no.136, 2008). It is argued that generous insurance schemes tend to increase the level of SA (Osterkamp \& Röhn, 2007), and the statistics on SA in Norway and Sweden echo this position: in the last decade, the mean rate of doctor-certified sickness absence in Norway was more than six per cent, but less than four per cent in Sweden (Statistics Norway 2011; Statistics Sweden 2011). Moreover, a survey in the Nordic countries five years ago has indicated profound differences in attitudes towards SA: the countries with the most restrictive benefits schemes are those with the most restrictive attitudes towards absenteeism due to sickness (Dahl et al., 2007). In brief, Norway and Sweden share many features, but sickness benefits are less generous in Sweden, the level of SA is substantially lower in Sweden, and Swedes have more restrictive attitudes towards SA. Contrasting experiences with SP among Norwegian and Swedish respondents, this study poses two research questions:

1. What is the distribution of SP in Norway and Sweden?

2. Which factors influence the frequency of SP in Norway and Sweden?

\section{Literature review}

Several studies have shown that over a 12-month period numerous employees have gone to work when they ought to have stayed at home for health reasons. A British study with close to 700 respondents indicated that more than 80 per cent of general practitioners, hospital physicians, and senior accountants engaged in SP. The respondents cited both cultural and organizational factors behind their decisions to not take sick leave, and among doctors one of the main barriers to taking sick leave was the difficulty of arranging cover (McKevitt et al., 1997). Approximately 80 per cent reported SP in a Norwegian study of more than 1000 physicians. Factors associated with 
SP include being of medium age (30-39 years) and having low job satisfaction, and the authors suggest that the latter correlation could be related to problems with taking sick leave (Rosvold \& Bjertness, 2001). In a study of 2400 female workers in lower-level elderly care, more than 70 per cent (Finland, Norway, and Sweden) and 80 per cent (Denmark) reported SP in the previous year. The main findings from this Nordic study are that more SA and SP accompanies increasing job stress, and that SP rises particularly in cases of high levels of job stress (Elstad \& Vabø, 2008). More than 70 per cent of the Danish core workforce reported one or more episodes of SP in a study with 13 000 respondents. Time pressure and a close relationship with colleagues increased the likelihood of SP. The analysis also showed that attitudes matter: respondents who found it more taxing to stay at home than to go to the workplace, being over-committed to work, and having a conservative attitude to absence were most likely to report SP (Hansen \& Andersen, 2008). More than 70 per cent had attended work even when they felt sick in a study of 200 respondents in a Canadian public-service organization. The top two reasons for SP were that 'others depend on me' and respondents had a 'high workload' (Caverley et al., 2007). In the Netherlands, about 60 per cent of a national sample of workers reported SP (Vroome, 2006). Finally, 50 per cent of the respondents in a Swedish labour force survey reported SP in 1997 (Aronsson et al., 2000), and in a study from 2000, the proportion was 70 per cent (Aronsson \& Gustafsson, 2005). In the latter study, the analysis of more than 3000 respondents indicated that having a health problem, a poor personal financial situation, difficulties in staff replacement, and time pressure were strong determinants of SP.

Aronsson (2012) presents the two main approaches to research on SP. First, European research has concentrated on the association between SP and health. Secondly, American research has investigated the consequences of SP on the productivity of organizations. With regard to the latter, it is claimed that SP causes much more aggregate productivity loss than SA, and that managing SP effectively could be a competitive advantage (Collins et al., 2005; Hemp, 2004). Although most studies agree that the total costs of SP exceed the total costs of SA, the remarkable variation in studies that report the effects of SP on productivity and the consequent costs is striking (Johns, 2010). Thus, the effects on the quantity and quality of the work performed by personnel practising SP should be subject to further investigation.

With regard to SP and health, there are some studies on the strongly positive association between absenteeism and presenteeism (Aronsson et al., 2000; Elstad \& Vabø, 2008; Hansen \& Andersen, 2008; Böckerman et al., 2010). Kristensen (1991) has argued that studying SP could enhance our understanding of SA because SP and SA are conclusions of the same decision process. Moreover, some studies have found that several episodes of SP during the previous year is a risk factor for future SA, and that SP can cause serious health problems at a later stage (Kivimäki et al., 2005; Bergström et al., 2009).

Various studies have also investigated what causes SP. They have explored the influence of personal factors and work-related factors on SP (Aronsson 2012). Personal factors that have been found to have an impact on SP include: state of health, education level, age, economic situation, body-mass index (BMI), social support, state of work-life balance, norms and feelings of 
moral obligation. The work-related factors that have been found to influence SP include: occupational affiliation, job security or insecurity, workplace culture, possibilities for staff replacement, work burden, working-time arrangements, employment in the public or private sector, job stress, job satisfaction, social support, control over work tasks, and potential for adjusting work demands (McKevitt et al., 1997; Rosvold \& Bjertness, 2001; Lovell, 2004; Aronsson et al., 2000; Aronsson \& Gustafsson, 2005; Elstad \& Vabø, 2008; Hansen \& Andersen, 2009; Agudelo-Suárez et al., 2010; Böckerman \& Laukkanen, 2010; Hansen 2010).

This article expands the body of work on SP. First, this article shows the distribution of SP in Norway and Sweden. There are two former studies on SP in Sweden (Aronsson et al., 2000; Aronsson \& Gustafsson, 2005), but SP in Norway has not previously been measured in a national sample. Secondly, this article uncovers relevant determinants for SP in both Norway and Sweden. In comparison with the previous literature, the analyses replicate some factors but also discuss the potential impact of 'new' factors, such as ethnic background, type of employment, position, and physical working conditions. Thirdly, there are few comparative studies that have discussed the determinants for SP in various countries (Aronsson, 2012). This article takes a comparative approach, asking whether the same factors influence SP both in Norway and in Sweden.

\section{Method}

The data are from responses to a postal questionnaire in Norway and Sweden. The questionnaire was sent to random samples of Norwegian and Swedish workers between 20 and 60 years of age. In both countries, the process of selecting the gross sample was simple random sampling from the population of workers between 20 to 60 years of age. The collection of data was done in 2011 and took two months, from the beginning of March to the beginning of May. It was done in accordance with the rules set by the committees for medical research ethics in both Norway and Sweden, and was approved by the Norwegian Social Science Data Services. The Research Council of Norway provided funding for the collection of data, but the Council had no role in the study design, in the collection, analysis, and interpretation of the data, or in the writing of the article.

The survey was one of three cross-country data collections in a research project called Social factors contributing to sickness absence (SOFAC). The age span of 20 to 60 was used in all data collections for SOFAC. We decided to exclude the oldest workers in the labour force from the sampling because people above 60 are often entitled to disability pensions and other support. Thus, there is a selective process at work, since aged workers with worst health retire early. Because health is an important determinant of both SP and $\mathrm{SA}$, it has likely a substantial impact on the distribution and determinants of SP among the oldest workers.

The net samples included 1600 Norwegians and 1250 Swedes. The response rate was only 33 per cent in both countries. Response rates tend to be very low for postal questionnaires (Edwards et al., 2002), but it was the only financially viable option for this cross-country study. To maximize the response rate, the length of the questionnaire was kept quite short (four pages and 60 
questions), a postal follow-up including a questionnaire was sent, the return envelope was pre-paid, and the information letter stressed the benefits of the study to society. In retrospect, there are a number of strategies that could have been considered: the use of monetary or non-monetary incentives, using personalized questionnaires and letters, contacting participants before sending the questionnaires, sending more than one follow-up, and using the official envelopes of Stockholm University and Lillehammer University College. Such strategies could have increased the response rate and improved the quality of our study (Heberlein \& Baumgartner 1978; Edwards et al., 2002).

To test for non-response bias, known values from the population of potential participants (workers between 20 and 60 years of age) were compared with the values that prevail in the subgroup that answered the questionnaire. It is positive that the Norwegian and Swedish net samples were representative with regard to ethnic background, as well as representative of regional dimensions like the size of municipality, county, and centrality/peripherality. The Norwegian net sample is representative with regard to gender, whilst there is an overrepresentation of women in the Swedish sample. In the net samples for Norway and Sweden, those in the age group 40-60 are overrepresented and those between 20 to 39 years are underrepresented. The data were weighed according to age and gender in order to remedy the underrepresentation of young workers and men.

More than 2500 respondents who were working, on parental leave, or absent due to illness (SA) answered the questions about SP. The survey was relevant for those currently on parental leave or SA, because they could have practised SP prior to the time of the survey (e.g., if they were on parental leave for the last two months, they still had 10 months to practise SP). The frequency of SP (the distribution of SP episodes) was measured by the following question: 'During the last 12 months, did you go to work despite feeling so ill that you should have taken sick leave?' The respondents were presented with four alternatives: 'No'; 'Yes, one to two times'; 'Yes, three to four times'; 'Yes, five or more times'. The length of SP (in days) was measured by this question: 'Please estimate how many days you went to work when you were feeling so ill that you should have taken sick leave during the last 12 months.' The respondents were presented with five alternatives: 'None'; '1-7 days'; '8-14 days'; ' $15-30$ days'; ' 31 days or more'. It might be objected that it may well measure a low threshold to report 'should have taken sick leave', but most of the studies referred to in the literature review use the same (or very similar) questions as this study does.

Answers to questions about the past can be imperfect and thereby affect the validity of the results of the survey. The responses to questions on SP might have been influenced by recall bias. The accuracy of recall depends on the time interval between the event and the time of its assessment. Ninety-eight per cent of the potential respondents answered questions about SP. It is hard to make conclusions about the accuracy of our survey, but the high number of responses is an indication that our respondents found the questions about SP to be relatively uncomplicated. It could be that our choice to give alternatives (i.e., closed questions) made it simpler for respondents to answer these questions about SP than it would have been if they were required to remember the exact number of days/episodes (i.e., open questions). 
Response bias refers to instances when a respondent intentionally responds incorrectly to a question about their personal history. Some studies have shown that employees tend to under-report their SA (van Poppel et al., 2002). It could be that data on SP suffer from under-reporting or over-reporting, but this study did not control for this possibility. It must be noted, though, that response bias is particularly problematic when you do interviews either faceto-face or over the phone. There was less of an interview effect in this study, since respondents wrote down their answers in an anonymous survey.

Three types of analysis are performed: cross-tabulations with the MannWhitney $U$ test, and binary and multinomial logistic regression on factors influencing SP. The Mann-Whitney $U$ test is a non-parametric test for assessing whether one of two samples of independent observations tends to have larger values than the other. Binary and multinomial logistic regression is used to detect which factors influence the frequency of SP.

The regression models include $11 \mathrm{x}$-variables and are quite complex. Still, the inclusion of other variables could have changed the results. Most importantly, the study does not include information about respondents' health, arguably an important determinant of SP. In addition, former empirical studies have reported substantial variation in the prevalence of SP by industry within the private sector. This article does not analyse the effects on industry with the exception that there is a set of indicators for the public and the private sectors.

\section{Frequency and length of sickness presenteeism}

Table 1 displays information about the distribution of SP episodes. Fifty-five per cent of the Norwegian respondents and 56 per cent of the Swedish respondents replied that at some point in the previous year they had gone to work despite feeling so ill that they should have taken sick leave. Results between the two countries are also similar when we investigate the number of SP episodes: 36 per cent of Norwegians and 38 per cent of Swedes report one or two episodes; ten per cent in both countries report three and four episodes; and nine per cent of Norwegians and eight per cent of Swedes report five or more episodes.

Table 1: Distribution of SP episodes in Norway and Sweden, percentage

\begin{tabular}{lcc}
\hline & Norway & Sweden \\
& & \\
0 episodes & 45 & 44 \\
1-2 episodes & 36 & 38 \\
3-4 episodes & 10 & 10 \\
5 or more episodes & 9 & 8 \\
Sum & 100 & 100 \\
\hline Mann Whitney: $p>0.05$ & &
\end{tabular}

SP has not been measured previously in a national sample in Norway, but results for Sweden are comparable to studies done a decade ago: 44 per cent report no SP in 2011 compared with 50 per cent in 1997 and 30 per cent in 
2005; 48 per cent report one to four SP episodes in 2011 compared with 42 per cent in 1997 and 55 per cent in 2000; and eight per cent report five or more SP episodes compared with eight per cent 1997 and 15 per cent in 2000 (Aronsson et al., 2000; Aronsson \& Gustafsson, 2005).

Table 2: Distribution of SP days in Norway and Sweden, percentage

\begin{tabular}{lcc}
\hline & Norway & Sweden \\
0 days & 45 & 44 \\
1-7 days & 37 & 43 \\
8-14 episodes & 11 & 9 \\
15-30 days & 4 & 3 \\
31 days or more & 3 & 1 \\
Sum & 100 & 100 \\
\hline Mann Whitney: $\mathrm{p}>0.05$ & &
\end{tabular}

Table 2 presents the length of SP. In the previous year, 43 per cent of Swedes and 37 per cent of Norwegians report one to seven days of SP, nine per cent and 11 per cent report eight to 14 days of SP, and four per cent of the Swedish and seven per cent of the Norwegian respondents report 15 or more days of SP. Although Norwegian respondents seem to report more days of SP than Swedes in the sample, the difference between the two groups is not statistically significant.

\section{Factors associated with sickness presenteeism}

This section assesses the impact of 11 variables on the frequency of SP episodes. For the sake of simplicity, the dependent variable has values 0 (no SP) and 1 (one or more SP episodes).

Gender, age, and ethnic background are important demographic factors. Some studies indicate that men more often practise SP (Hansen \&Andersen, 2009; Böckerman Laukkanen, 2010), whilst other studies reveal small or no gender differences (Aronsson et al., 2000; Agudelo-Suárez et al., 2010). Similarly, some studies indicate that the older part of the workforce practises SP less often than younger workers do (Hansen \& Andersen, 2009), whilst other studies find no such correlation (Böckerman Laukkanen, 2010). Earlier studies in Norway and Sweden have shown that non-western immigrants have poorer health and higher rates of absence than natives and western immigrants (Nilsson, 2005; Bengtsson, 2006; Dahl et al., 2010). Ethnic background is seldom considered in discussions about SP, but a Spanish study has concluded that immigrant workers report more SP than their Spanish counterparts (Agudelo-Suárez et al., 2010).

Income and education are important measures of socio-economic position. It is reasonable to assume that personal income has an impact on SP; those with high income report less SP than those with low income (Aronsson et al., 2000; Hansen \& Andersen, 2009). The results with regard to education differ: in some studies people with less education report more SP than those who are 
highly educated (Aronsson \& Gustafsson, 2005; Böckerman Laukkanen, 2010), and other studies indicate that education has little explanatory power (Aronsson et al., 2000).

This study also included three work-related factors: employment type, position, and physical work. Previous studies have indicated that self-employed people are more likely to report SP than employees (Hansen \& Andersen, 2008; 2009), and that persons employed in public sector are more likely to be present than those employed in private companies (Aronsson et al., 2000; Bergström et al., 2009). In Sweden, a recent report in the series Social Insurance Report (Socialförsäkringsrapport) points out that managers have less absence compared with other groups of employees (Mulder, 2011). Could it be that managers are more likely to 'choose' SP over SA than employees without managerial responsibilities? Finally, this study investigated the relation between the physical working environment and SP. Previous studies of SA have shown that lifting, carrying, pushing or pulling loads, or any of these, comprises the factors increasing the risk of absence (Lund et al., 2006), and thus physical work should correlate with SP. The study by Hansen and Andersen (2009) indicates that work involving heavy lifting increases SP, and in the study by Aronsson et al. (2000), excess rates of SP were found among occupational groups characterized by (fairly) heavy physical work.

To summarize, this study included the following independent variables:

- Gender: male (reference category) and female

- Age in years

- Ethnic background: natives and western immigrants (comprising Western Europe, Canada, USA, Australia, and New Zealand) (reference category) and non-western immigrants (other countries)

- Education: high educational attainment (Bachelor degree or higher) (reference category) and low educational attainment (upper secondary school and lower)

- Income: low income (gross annual income of 299,000 NOK/SEK or less) medium income (gross annual income between 300,000 and $499,000 \mathrm{SEK} / \mathrm{NOK}$ ), and high income (gross annual income of $500,000 \mathrm{NOK} / \mathrm{SEK}$ or more) (reference category)

- Type of employment: employee in private sector, employee in public sector, and self-employed (reference category)

- Position: executive, middle management, and non-management (reference category)

- Physical work: sedentary work (reference category) and physical work

Table 3 presents unstandardized coefficients (B), odds ratios (OR), and pvalues $\left(^{*}\right)$ from binary logistic regression analyses of SP episodes in Norway and Sweden. $B$ refers to the average increase in the dependent variable $(Y)$ with one measurement increase in the independent variable $(X)$. P-value refers to the probability that $X$ and $Y$ are not related. OR is a calculation of the probability of the outcome event occurring divided by the probability of the event not occurring.

In both models, there are statistically significant effects $(p<0.05)$ for 'low income', 'medium income', 'middle management', and 'physical work'. In 
addition, 'non-western immigrant', 'low education', 'employee in public sector' and 'employee in private sector' are significant in the Norwegian model, whilst 'executive' is significant in the Swedish model. The remaining variables are non-significant.

Table 3: Binary logistic regression of the factors influencing SP in Norway and Sweden.

\begin{tabular}{lcccc}
\hline \multicolumn{1}{c}{ Variables } & \multicolumn{2}{c}{ Norway } & \multicolumn{2}{c}{ Sweden } \\
& B & OR & B & OR \\
Constant & $-1.00^{\star}$ & 0.37 & -0.31 & 0.73 \\
Female & 0.03 & 1.03 & -0.19 & 0.83 \\
Age & -0.00 & 1.00 & -0.00 & 1.00 \\
Non-western immigrant & $1.68^{\star \star}$ & 5.37 & 0.03 & 1.03 \\
Low education & $0.33^{\star}$ & 1.40 & 0.14 & 1.15 \\
Low income & $0.54^{\star *}$ & 1.72 & $0.53^{\star}$ & 1.70 \\
Medium income & $0.30^{\star}$ & 1.35 & $0.65^{\star}$ & 1.91 \\
Employee in public sector & $0.44^{\star}$ & 1.56 & -0.11 & 0.90 \\
Employee in private sector & $0.67^{\star *}$ & 1.95 & 0.03 & 1.03 \\
Executive & 0.35 & 1.44 & $0.69^{\star}$ & 1.99 \\
Middle management & $0.43^{\star *}$ & 1.54 & $0.40^{\star}$ & 1.49 \\
Physical work & $0.37^{\star *}$ & 1.44 & $0.35^{\star}$ & 1.41 \\
\hline
\end{tabular}

${ }^{* *}=$ Significant at $0.01,{ }^{*}=$ Significant at 0.05

Reference categories: male; native or western immigrant; high education; high income; self-employed; non-management; sedentary work Nagelkerke $R^{2}: 0.07$ (Norway) and 0.04 (Sweden)

ORs present an additional way of interpreting results. When the $O R$ is close to 1 , there is no particular effect; the higher it is over 1 , the stronger the positive effect; and the closer it is to 0 , the stronger the negative effect. Some results correspond: those with low income are 1.7 times more likely to report SP compared with those with high income; middle managers are 1.5 times more likely to report SP compared with those without managerial responsibilities; and persons with physical work are 1.4 times more likely to report SP than persons with sedentary work. The positive impacts on SP of both medium income and executive position are strongest in the Swedish model, and executives are 2.0 times more likely to report SP than those with no managerial responsibilities. The impacts of low education, whether one is an employee in the public sector or the- private sector, and non-western immigrant are strongest in the Norwegian model, and non-western immigrants are 5.4 times more likely to report SP than natives and western immigrants.

Multinomial logistic regression has been used to evaluate the results presented in table 3 further. Table 4 discerns between '0 SP episodes', '1-2 SP episodes', and ' $3+$ SP episodes'; '0 SP episodes' is the reference category. Most of the results in table 4 are similar to those in table 3 . Starting with the Norwegian model, we find statistically significant effects for non-western immigrants, middle management, and physical work (both categories of SP episodes); low education, employee in public sector, employee in private 
sector (1-2 SP episodes); low income and medium income (3+ SP episodes). In the Swedish model, physical work is significant at 1-2 SP episodes, whilst low income, medium income and executive are significant at $3+$ episodes.

Table 4: Multinomial logistic regression of the factors influencing SP in Norway and Sweden.

\begin{tabular}{|c|c|c|c|c|}
\hline \multirow[t]{2}{*}{ Variables } & \multicolumn{2}{|c|}{ Norway } & \multicolumn{2}{|c|}{ Sweden } \\
\hline & B & OR & B & OR \\
\hline \multicolumn{5}{|l|}{ 1-2 episodes of SP } \\
\hline Intercept & $1.38^{\star \star}$ & & -0.64 & \\
\hline Female & 0.01 & 1.01 & -0.09 & 0.92 \\
\hline Age & -0.00 & 1.00 & -0.00 & 1.00 \\
\hline Non-western immigrant & $1.63^{\star \star}$ & 5.10 & -0.20 & 0.82 \\
\hline Low education & $0.33^{*}$ & 1.39 & 0.23 & 1.25 \\
\hline Low income & 0.28 & 1.32 & 0.13 & 1.13 \\
\hline Medium income & 0.18 & 1.20 & 0.48 & 1.62 \\
\hline Employee in public sector & $0.60^{*}$ & 1.83 & 0.09 & 1.09 \\
\hline Employee in private sector & $0.82^{\star \star}$ & 2.28 & 0.16 & 1.17 \\
\hline Executive & 0.28 & 1.32 & 0.61 & 1.84 \\
\hline Middle management & $0.41^{\star *}$ & 1.50 & 0.34 & 1.40 \\
\hline Physical work & $0.28^{\star *}$ & 1.32 & $0.38^{\star}$ & 1.47 \\
\hline \multicolumn{5}{|l|}{$3+$ episodes of SP } \\
\hline Intercept & $-2.26^{\star \star}$ & & $-1.75^{\star \star}$ & \\
\hline Female & 0.06 & 1.07 & -0.40 & 0.67 \\
\hline Age & -0.00 & 1.00 & -0.00 & 1.00 \\
\hline Non-western immigrant & $1.77^{\star \star}$ & 5.88 & 0.37 & 1.44 \\
\hline Low education & $0.35^{*}$ & 1.42 & -0.03 & 0.97 \\
\hline Low income & $1.01^{\star *}$ & 2.76 & $1.47^{\star *}$ & 4.33 \\
\hline Medium income & $0.56^{\star}$ & 1.76 & $0.86^{\star}$ & 2.35 \\
\hline Employee in public sector & 0.21 & 1.24 & -0.46 & 0.63 \\
\hline Employee in private sector & 0.45 & 1.56 & -0.16 & 0.85 \\
\hline Executive & 0.50 & 1.64 & $0.86^{*}$ & 2.35 \\
\hline Middle management & $0.47^{\star}$ & 1.60 & $0.54^{\star}$ & 1.71 \\
\hline Physical work & $0.53^{*}$ & 1.70 & 0.27 & 1.31 \\
\hline
\end{tabular}

Reference category for dependent variable: 0 episodes of SP

Reference categories for independent variables: male; native or western immigrant; high education; high income; working full time; self-employed; non-management; sedentary work

Nagelkerke $R^{2}: 0.08$ (Norway) and 0.06 (Sweden)

\section{Discussion}

This study of Norwegian and Swedish workers suggests that some SP during a working year might be more common than no SP. This finding is in accordance with prior studies of SP at the national level (Aronsson et al., 2000; Vroome, 2006; Hansen \& Andersen, 2009). An investigation of Swedish studies shows that the proportion of respondents reporting SP in 2011 was somewhat higher than it was in 1997, but lower compared with the remarkable upswing in SP in the study from 2000 (Aronsson et al., 2000; Aronsson \& Gustafsson, 2005). The fact that so many people report SP should make us 
aware of the chance that the current levels of SA in Norway and Sweden are lower than they would be if workers called in sick every time they felt ill. At this point, the reader would do well to note two main objections against the validity of self-reported SP. One is that there is the potential for respondents to overestimate SP, because it is plausible for people to over-report SP in order to appear more desirable in the eyes of others. The other objection raises questions about the severity of the illness, because it is worthwhile to consider whether people who report SP suffer from genuine illness.

This study has replicated many findings from former studies on factors associated with SP. Income level is important in both countries: workers with low and medium income more often report SP compared with those with high income. This connection resembles the previous Scandinavian studies, and Aronsson et al. $(2000,503)$ propose that the cause could be that low-paid persons have narrower personal financial margins compared with persons with high income: 'The financial loss of being absent from work has a greater impact on the low paid, and accordingly their disposition to presenteeism might be expected to be higher.'

The strong connection between SA and SP indicates that they are conclusions of the same decision-making process (Kristensen, 1991; Hansen \& Andersen, 2008). Managers generally have low levels of SA, and this could be interpreted as a reflection of their good health. At the same time, our results indicate that Norwegians and Swedes with managerial responsibilities report SP more often than employees without such responsibilities. Thus, it could be that managers do not have better health, but are more reluctant to partake in SA because they feel obligated to be at work, or for the love of the job or for some other reason.

In both countries, persons with physical work report SP more often than persons doing sedentary work. This result matches Hansen and Andersen's (2009) finding about the connection between heavy lifting and SP, and the analysis of SP among occupational groups made by Aronsson et al. (2000). In the latter study, the risk of SP was very low among 'deskbound' occupations (e.g., therapists, computing professionals, secretaries, numerical clerks, bookkeepers, business professionals) and very high among persons in occupations associated with (fairly) heavy physical work (e.g., day-care staff, teachers in primary school, nursing-home aides, nursing and midwifery professionals, different types of welfare workers). Occupational groups with high SP are characterized by interrelations with vulnerable groups such as the sick, the old, the young and small children, and ties created between the worker and their care recipients/pupils/clients might reduce the disposition to be absent from work and to increase SP (ibid.).

The most notable difference between Norway and Sweden involves ethnic background and SP. In Norway, non-western immigrants report SP more often than Norwegians and western immigrants, whilst in Sweden there is no significant difference between non-western immigrants and western immigrants and Swedes. Previous research shows that non-western immigrants have poorer health, precarious employment in semi-skilled and low-paid jobs in the service sector, and higher absence rates than natives and western immigrants (Timonen, 2004; Nilsson, 2005; Bengtsson, 2006; Dahl et al., 2010). In the Norwegian sample for this study, more SP among non- 
western immigrants is observed, but not more SA. This may reflect that nonwestern immigrants have poorer health, but feel compelled to turn up at work instead of staying at home when ill. Other possible causes for more SP among non-western immigrants include lack of knowledge of social benefits, difficulties navigating the health system, and cultural factors (Agudelo-Suárez, 2010). It could be that different perceptions of illness exist between native and foreign-born workers. Or it might be that some non-western immigrants have particular work motivations; for instance, it is likely that workers are more prone to SP if their goal is to earn as much as possible in the shortest possible time. This study lacks the capability to trace causal relationships, and one is simply left to suggest causes for higher presenteeism among non-western immigrants. Furthermore, it is difficult to explain the notable difference between results for Norway and Sweden: more research is needed to follow up on the missing correlation between ethnic background and SP in Sweden.

The correlations between type of employment and SP raise questions about former studies. In this study, employees in the private sector report SP most often, followed by employees in the public sector, and then the self-employed. This ranking is the opposite of that expected from former research (Bergström et al., 2009; Hansen \& Andersen, 2008)

Finally, gender and age are insignificant in both countries. Previous studies have shown tendencies of age and gender differences, but age and gender have relatively little explanatory value with regard to SP (Aronsson et al. 2000). Education matters in Norway, but not in Sweden. In the former studies, there is no clear pattern with regard to education. In some studies people with less education report more SP than those who are highly educated (Aronsson \& Gustafsson, 2005; Böckerman Laukkanen, 2010), whilst education is less relevant in other studies (Aronsson et al., 2000; Elstad \& Vabø, 2008).

\section{Conclusion}

Knowledge of the distribution of and determinants for SP is still relatively sparse, and this article provides some insight into these matters. Still, the results presented must be interpreted with caution since the response rate is low and the responses to SP might suffer from recall bias.

The article has raised two research questions. The first question raised was that of the distribution of SP in Norway and Sweden. This study of Norwegian and Swedish workers suggests that some SP during a working year may be more common than no SP, and this is in accordance with former studies of SP at the national level (Aronsson et al., 2000; Aronsson \& Gustafsson, 2005; Vroome, 2006; Hansen \& Andersen, 2009). The frequency of SP episodes is similar in the two countries (approximately 45 per cent report no SP, 35 per cent report one or two SP episodes, and 20 per cent report three or more SP episodes). Norwegians in the sample report longer SP than Swedes in the sample (18 per cent of Norwegians report 8 days or more SP compared with 13 per cent of the Swedish respondents), but the difference between the two groups is not statistically significant.

The second question was about which factors influence the frequency of SP in Norway and Sweden. Comparative studies are useful since they put results for each country in a broader context. In both countries, respondents with low and 
medium income, employment, managerial responsibilities, and physical work are more likely to report SP. Executives are overrepresented with SP in Sweden, whilst non-western immigrants and the less educated are overrepresented with SP in Norway. The influences of both gender and age are very small in both countries.

The use of self-reported and cross-sectional data means that results from this study are tentative. Several issues pertaining to SP remain to be studied. More than half of the workers in the study practised SP in the previous year, but we know neither the symptoms that people who practise SP refer to nor whether they suffer from genuine illnesses. Further research, especially qualitative research, is needed to understand and explain the differences with regard to the determinants between the two countries, as well as the consequences of $\mathrm{SP}$ in both shorter and longer terms.

\section{References}

Agudelo-Suárez, A.A, Benavides, F.G., Felt, E, Ronda-Pérez, E., Vives-Cases, C., \& García, A.M. (2010). Sickness presenteeism in Spanish-born and immigrant workers in Spain. BMC Public Health, 10:791

Aronsson, G. (2012). Sjuknärvaro: en forskningsöversikt [Sickness presence: a research review]. In Alexanderson, K. (ed.). Socialförsäkringsforskning. En vänbok till Staffan Marklund [Social-Insurance Research: a liber amicorum to Staffan Marklund]: 23-33. Stockholm: Karolinska Institute

Aronsson, G., Gustafsson K., \& Dallner M. (2000). Sick but yet at work: An empirical study of sickness presenteeism. Journal of Epidemiology and Community Health, 54:502-509.

Aronsson, G., \& Gustafsson, K. (2005). Sickness presenteeism: prevalence, attendance-pressure factors, and an outline of a model for research. Journal of Occupational Environmental Medicine, 47:958-966.

Bengtsson, T. \& Scott, K. (2006) Immigrant consumption of sickness benefits in Sweden, 1982-1991. Journal of Socio-Economics, 35: 440-457.

Berge, C. (2012) Internasjonal sammenligning av sykefravær [International comparison of sickness absence]. Økonomiske analyser 2:24-29.

Bergström, G., Bodin, L., Hagberg, J., Aronsson, G., Josephson, M. (2009). Sickness presenteeism today, sickness absenteeism tomorrow? A prospective study on sickness presenteeism and future sickness absenteeism. Journal of Occupational and Environmental Medicine, 51:629-638.

Böckerman, P. \& Laukkanen, E. (2010). What makes you work while you are sick? Evidence from a survey of workers. European Journal of Public Health, 20:4346.

Caverley, N., Cunningham, J.B., \& MacGregor, J.N. (2007). Sickness presenteeism, sickness absenteeism, and health following restructuring in a public service organization. Journal of Management Studies, 44: 304-319. 
Collins, J.J., Baase, C.M., Sharda, C.E. Ozminkowski, R.J., Nicholson, S., Billotti, G.M., Turpin, R.S., Olson, M., \& Berger, M.L. (2005). The assessment of chronic health conditions on work performance, absence, and total economic impact for employers. Journal of Occupational and Environmental Medicine, 47: 547-557.

Dahl, S.Å., Hansen, H.T., \& Olsen, K.M. (2010). Sickness absence among immigrants in Norway, 1992-2003. Acta Sociologica, 53: 35-52.

Dahl, S.Å., Holmås, T.H., \& Skjeret, F. (2007). En kartlegging av holdninger til sykefravær $i$ Norden [Attitudes towards sickness absence in the Nordic countries]. Oslo: Institute for Research in Economics and Business Administration.

Edwards, P., Roberts, I., Clarke, M., DiGuiseppi, C., Pratap, S., Wentz, R., \& Kwan, I. (2002). Increasing response rates to postal questionnaires: systematic review. BMJ 324:1183

Elstad, J.I. \& Vabø, M. (2008). Job stress, sickness absence and sickness presenteeism in Nordic elderly care. Scandinavian Journal of Public Health, 36: 467-74.

Government proposition no.136 (2008). En reformerad sjukskrivningsprocess för ökad återgång $i$ arbete [ $\mathrm{A}$ reformed sick leave for increased return to work]. Stockholm: Ministry of health and social affairs.

Hansen, C.D. (2007). 'Making a virtue' of going ill to work - reflections on the necessity of everyday workplace 'suffering'. Tidsskrift for Forskning i Sygdom og Samfund, 7:69-88.

Hansen, C.D., \& Andersen, J.H. (2008). Going ill to work - what personal circumstances, attitudes and work-related factors are associated with sickness presenteeism? Social Science \& Medicine, 67:956-964

Hansen, C.D. \& Andersen, J.H. (2009). Sick at work - a risk factor for long-term sickness absence at a later date? Journal of Epidemiology and Community Health, 63:397-402.

Heberlein, T.A., \& Baumgartner, R. (1978). Factors affecting response rates to mailed questionnaires: A quantitative analysis of the published literature. American Sociological Review 43: 447-462.

Hemp, P. (2004). Presenteeism: At work - But out of it. Harvard Business Review 82: 49-58.

Johns, G. (2010). Presenteeism in the workplace: A review and research agenda. Journal of Organizational Behavior, 31: 519-542.

Kivimäki M., Head, J., Ferrie, J.E., Hemingway, H., Shipley, M.J., Vahtera, J., \& Marmot, M.G. (2005). Working while ill as a risk factor for serious coronary events: the Whitehall II study. American Journal of Public Health, 95: 98-102.

Kristensen, T. (1991). Sickness absence and work strain among Danish slaughterhouse workers: an analysis of absence from work regarded as coping behaviour. Social Science \& Medicine, 32: 15-27.

Lovell, V. (2004). No time to be sick: Why everyone suffers when workers don't have paid sick leave. Washington, DC: Institute for Women's Policy Research.

Lund, T., Labriola, M., Christensen, K.B., Bültmann, U., \& Villadsen, E. (2006). Physical work environment risk factors for long term sickness absence: prospective findings among a cohort of 5357 employees in Denmark. BMJ 332: 449-452. 
McKevitt, C., Morgan, M., Dundas, R., \& Holland, W.W. (1997). Sickness absence and 'working through' illness: a comparison of two professional groups. Journal of Public Health Medicine 19: 295-300.

Ministry of Labour and Social Inclusion (2011). The Norwegian Social insurance scheme 2011. Oslo: Ministry of labour and social inclusion.

Mulder, M. (2011). Sjukskrivningsdiagnoser i olika yrken [Sick-leave diagnoses in different occupations]. Stockholm: The Swedish Social Insurance Agency.

Nilsson, M. (2005). Differences and similarities in work absence behaviour. Empirical analyses of micro panel data. PhD Thesis. Växjö: Växjö University.

Osterkamp, R., \& Röhn, O. (2007). Being on sick leave: Possible explanations for differences of sick-leave days across countries. CESifo Economic Studies, 53: 97-114

Rosvold, E.O., \& Bjertness, E. (2001). Physicians who do not take sick leave: hazardous heroes? Scandinavian Journal of Public Health, 29: 71-75.

Statistics Norway (2011). Working conditions, sickness absenteeism. Retrieved from http://www.ssb.no/english/subjects/06/02/

Statistics Sweden (2011). Labour market, Retrieved from http://www.scb.se/Pages/SubjectArea__2441.aspx

Timonen, V. (2004). New risks - are they still new for the Nordic welfare states. In Taylor-Gooby, P. (ed.). New risks, new welfare. The transformation of the European welfare state: 83-110. Oxford: Oxford University Press.

van Poppel, M., de Vet, H., Koes, B., Smid, T., \& Bouter, L.M. (2002). Measuring sick leave: a comparison of self-reported data on sick leave and data from company records. Occupational Medicine, 52: 485-490.

Vingård, E., Alexanderson, K., \& Norlund, A. (2004). Chapter 10. Sickness presence. Scandinavian Journal of Public Health, 32: 216-221.

Vroome, E. (2006). Prevalence of sickness absence and 'presenteeism'. Retrieved from http://eurofound.europa.eu/ewco/2006/07/NL0607019l.htm 